\title{
Cardiac implantable electronic device lead extraction in patients with underlying infection using open thoracotomy or percutaneous techniques
}

\author{
Divyang Patel, Faris Khan, Hemal Shah, \\ Sanjoy Bhattacharya, Evan Adelstein, Samir Saba
}

Heart and Vascular Institute, University of Pittsburgh Medical Center, Pittsburgh, PA, United States

\begin{abstract}
Background: Explanting infected cardiac implantable electronic devices (CIEDs) and extracting their associated leads can be performed percutaneously (EP) or via open-thoracotomy (OR) approach. In this study, we examined the characteristics and outcomes of infected CIED patients undergoing EP vs. OR extraction procedures.

Methods: All patients (EP: $n=329$ and OR: $n=24$ ) who received lead extraction in the presence of an infected CIED from 2005 to 2010 at the University of Pittsburgh Medical Center were included in this study. Demographic and clinical characteristics were obtained from the electronic medical records. The Charlson comorbidity index (CCI) was used to adjust for severity of co-morbid conditions.

Results: Compared to the EP group, OR patients were more likely to have positive blood cultures, larger vegetations, and worse CCI scores. They also had higher total mortality rates at $1(p=0.036), 6(p=0.020)$, and 12 months $(p=0.012)$ after the procedure. One-year survival after lead extractions was significantly better for the EP compared to the OR group $(p=0.002)$ even after adjusting for other comorbid illnesses $(H R=2.6, p=0.010)$ in a Cox regression model. Conclusions: Infected CIED patients undergoing open-chest lead extraction are sicker and have higher mortality rates compared to those undergoing percutaneous extraction. Randomized, prospective data are needed to determine whether the procedural strategy for lead extraction accounts in part for the difference in outcome. (Cardiol J 2015; 22, 1: 68-74)
\end{abstract}

Key words: cardiac implantable electronic devices, infection, lead extraction, thoracotomy

\section{Introduction}

Multicenter, randomized studies have consistently demonstrated the value of implantable cardioverter-defibrillators in reducing mortality in patients with chronic heart failure and low left ventricular ejection fraction as well as in patient with spontaneous or inducible ventricular arrhythmias [1-3]. This has led to a dramatic expansion of cardiac implantable electronic devices (CIEDs) indications $[4,5]$. As more patients received CIEDs, the number of complications associated with these procedures has increased [6] including CIED infections which are rising faster than implantation rates

Address for correspondence: Samir Saba, MD, Chief of Cardiac Electrophysiology, University of Pittsburgh Medical Center, 200 Lothrop Street, UPMC Presbyterian, Suite B-535, Pittsburgh, PA 15213-2582, USA, tel: (412) 6476272 ,

fax: (412) 647 7979, e-mail: sabas@upmc.edu

Received: 31.01.2014 Accepted: 11.04.2014 
[7-9] and which necessitate complete removal of infected CIEDs [10-13]. Traditionally, lead extraction in patients with large vegetations has been performed using open thoracotomy (OR), instead of percutaneous (EP) techniques, in order to minimize the risk of embolization $[14,15]$. Recently, however, several studies have shown that lead extraction in patients with infected leads and large vegetations can be safely done via $\mathrm{EP}$ techniques [16-18]. Furthermore, OR may be associated with higher rates of significant adverse events that can affect mortality [12]. We therefore examined the outcome of patients undergoing lead extraction for infectious indications by OR or EP techniques, focusing on total mortality after the procedure.

\section{Methods}

All research methods for this study were approved by the University of Pittsburgh Internal Review Board. All patients ( $\mathrm{n}=353$ ) who received lead extraction in the presence of an infected CIED at one of the hospitals of the University of Pittsburgh Medical Center from January 2005 to December 2010 were included in this study. Detailed demographic and clinical characteristics for all the patients were obtained from the electronic medical record. Procedural details were retrieved from the electrophysiology and cardiothoracic surgery operative notes.

Device infections were defined by the treating physician using clinical symptoms of infection such as fever, swelling, redness, pain, exposure of the device or leads at the device implantation site, or positive blood cultures. Using the Duke's criteria for endocarditis, presence, size, and location of vegetations were documented by echocardiography. Patients were selected to undergo open-chest vs. closed-chest lead extraction after discussion consensus agreement between the treating electrophysiologist and the cardiothoracic surgeon. In these discussions, the risk of open-chest surgery was weighed against the risk of embolization of large vegetations during closed-chest surgery. If the patient required additional surgery including valve repair or coronary artery bypass grafting, the decision naturally favored the open surgery approach.

Prior to the procedures, patients were treated with intravenous antibiotics. The OR procedures were performed by 11 cardiothoracic surgeons, whereas the EP procedures were performed by 5 electrophysiologists. Those who received OR extractions were endotracheally intubated and given general anesthesia. Through a median ster- notomy, access to the heart was obtained. Patients were heparinized and placed on cardiopulmonary bypass. The leads were dissected, keeping control over vegetations when present, to prevent embolization. Patients undergoing EP laboratory procedures had a $5 \mathrm{Fr}$ femoral arterial line placed for continuous hemodynamic monitoring as well as a $7 \mathrm{Fr}$ femoral venous sheath for central venous access. If indicated, a temporary pacemaker wire was placed from the femoral or jugular area. Intravenous deep sedation was used in all cases. The distal helices of leads were retracted when possible and traction was applied at the beginning of each case. If extraction was unsuccessful with traction alone, the leads were then cut, sized, and fitted with a locking stylet (Spectranetics Inc., Colorado, Springs, CO). A 12 Fr, 14 Fr, or 16 Fr laser-powered sheath system (Spectranetics Inc., Colorado Springs, CO) was used depending on the lead size and the operator's choice. At the end of the procedure, the CIED pocket was closed with suture material or packed and left to heal by secondary intention, as clinically indicated. All EP procedures had cardiothoracic surgical back-up available on site. All patients had $4 \mathrm{U}$ of packed red blood cells on standby in case of the need for emergent transfusion.

The primary outcome of interest for the present study was total mortality at 1,6 , and 12 months following the index procedure. Other procedure-related complications of lead extraction were also assessed using the definitions previously established in the consensus manuscript on lead extractions published in 2009 [19].

\section{Statistical analysis}

All continuous variables are presented as mean \pm \pm standard deviation. All categorical variables are presented as absolute numbers and percentages. Comparison of means was performed using the Student's t-test. Comparison of categorical variables was performed using Fisher's exact test. Survival curves were constructed using the Kaplan-Meier method and inter-group comparison was assessed using the log rank test. Independent predictors of the time to death were examined using the Cox proportional hazards test. In order to correct for baseline differences in overall clinical status, the Charlson comorbidity index (CCI) was used, taking into account the presence and severity of 19 comorbid conditions that impact mortality [2]. A 2 -sided p-value $\leq 0.05$ was considered statistically significant. All analyses were performed on PAWS software version 19.0 (IBM, Armonk, NY, USA). 


\section{Results}

A total of 353 patients who underwent lead extraction were included in this study (24 in the OR group and 329 in the EP group). Baseline characteristics for patients receiving lead extraction via OR or EP techniques are shown in Table 1. Patients undergoing lead extraction via OR were more likely to have positive blood cultures, vegetations on the leads or other heart valves, and larger size vegetations. Their CCI was also significantly higher, indicating a worse overall clinical condition. All other clinical characteristics were similar between the two study groups.

Of the 129 patients who had positive blood cultures, more than two-thirds had coagulase-negative or coagulase-positive Staphylococcal infections. As shown in Figure 1, the breakdown by type of bacteria recovered from blood cultures was similar between the two study groups.

Table 2 shows the mortality for the two study groups at 1,6 , and 12 months after the index procedure, as well as the rates of non-fatal procedural complications. Using univariate analysis, patients in the OR group had significantly higher total mortality rates at 1,6 , and 12 months after the index procedure, compared to the EP group. There were no intraoperative deaths in either one of the two study groups.

The cause of death could be ascertained for all but 2 patients who expired within 30 days of the index procedure (4 deaths in OR group and 15 deaths in EP group). Of the 4 (17\%) mortalities in the OR group, 3 patients died of cardiogenic shock, while 1 patient died of a massive stroke. Of the 15 (5\%) mortalities in the EP group, 6 died of severe hypotension attributed to sepsis (1 with confirmed septic embolization), 5 suffered multi-organ failure (1 patient refusing dialysis therapy for kidney failure), and 2 patients died as a result of cardiac arrest consisting of ventricular arrhythmia and asystole, respectively. For the remaining $2 \mathrm{EP}$ cases, the mode of death could not be ascertained.

There were no significant differences in the non-fatal complication rates experienced during OR vs. EP extraction procedures up to 30 days after the index procedure. Intraoperatively, there were no complications reported in the OR group compared to 6 in the EP group (significant blood loss requiring transfusion: $\mathrm{n}=3$, atrial lead dislodgement: $n=1$, and fractured lead with retained fragment: $\mathrm{n}=2$ ). Non-lethal complications within 30 days of the index procedure were also examined for the two study groups. There were
$2(8 \%)$ non-lethal complications in the OR group compared to $16(5 \%)$ in the EP group. Both OR cases involved chest bleeding requiring surgical exploration. Of the $16 \mathrm{EP}$ complications, 8 involved hematoma formation at the site of device explantation requiring evacuation in the electrophysiology laboratory within 5 days of the index procedure. The other 8 complications included septicemia $(n=1)$, hemothorax $(n=1)$, atrial lead dislodgement at reimplantation $(\mathrm{n}=1)$, pulmonary embolism $(n=1)$, pneumothorax $(n=1)$, and intra-operative complications $(n=3)$ as mentioned above.

Table 3 shows the characteristics of patients who died vs. those who were alive at 1 year after the index procedure. Univariate predictors of death at 1 year after the index procedure included older age, the presence of positive blood cultures, presence of diabetes mellitus, presence of coronary artery disease, higher baseline serum creatinine level, lower baseline ejection fraction, a higher CCI, and performing lead extraction via OR as opposed to via EP approach. There was also a trend towards an association between female gender and death at 1 year after the index procedure. As expected, the presence of coronary disease predicted lower ejection fraction and the presence of diabetes mellitus predicted higher serum creatinine level.

The time to death in the two study groups was also examined. As shown in Figure 2, patients treated with EP lead extractions had significantly better survival than those treated with $\mathrm{OR}(\mathrm{p}=0.002)$. After adjusting for age, gender, and CCI using multivariate Cox proportional hazard analysis, performing lead extraction through OR remained an independent predictor of death (hazard ratio $=$ $=2.60,95 \%$ confidence interval $1.26-5.37, \mathrm{p}=0.010$; Table 4).

\section{Discussion}

Our study reveals that in cases of CIED infection, lead extraction using closed-chest EP techniques is associated with lower mortality rates at 1,6 , and 12 months and better 1 -year survival compared to the OR approach. This finding is however tampered by the fact that the OR population was significantly sicker than the EP group. In this study, we also report on the mode of death of patients who expired within 30 days of the index procedure.

There is a paucity of studies in the literature addressing the death rate and cause of death from lead extraction in the case of CIED infections. Our study included 24 patients receiving OR for infec- 
Table 1. Patient demographics.

\begin{tabular}{|c|c|c|c|}
\hline Variable & $\begin{array}{l}\text { Open thoracotomy } \\
\qquad(n=24)\end{array}$ & $\begin{array}{c}\text { Percutaneous } \\
\text { techniques }(n=329)\end{array}$ & $\mathbf{P}$ \\
\hline Age [years] & $68 \pm 11$ & $70 \pm 14$ & 0.54 \\
\hline Male & $79 \%$ & $75 \%$ & 0.60 \\
\hline Implantable cardioverter-defibrillator explanted & $50 \%$ & $52 \%$ & 0.85 \\
\hline Oldest lead dwell time [months] & $55 \pm 55$ & $56 \pm 57$ & 0.96 \\
\hline QRS duration [ms] & $135 \pm 38$ & $135 \pm 40$ & 0.98 \\
\hline Ejection fraction [\%] & $36 \pm 15$ & $39 \pm 16$ & 0.57 \\
\hline Creatinine $[\mathrm{mg} / \mathrm{dL}]$ & $1.9 \pm 1.4$ & $1.5 \pm 1.1$ & 0.06 \\
\hline Coronary artery disease & $67 \%$ & $65 \%$ & 0.90 \\
\hline Diabetes & $50 \%$ & $37 \%$ & 0.20 \\
\hline Positive blood culture & $87 \%$ & $34 \%$ & $<0.001$ \\
\hline Vegetation present on the leads & $92 \%$ & $19 \%$ & $<0.001$ \\
\hline Size of vegetation $\left[\mathrm{cm}^{2}\right]$ & $4.5 \pm 2.5$ & $1.8 \pm 2.0$ & 0.001 \\
\hline $\begin{array}{l}\text { Need for concomitant heart surgery: } \\
\text { Mitral valve surgery }(n=4) \\
\text { Aortic valve surgery }(n=3) \\
\text { Tricuspid surgery }(n=1) \\
\text { Coronary bypass }(n=4) \\
\text { Atrial septal defect closure }(n=4)\end{array}$ & $67 \%$ & $0 \%$ & $<0.001$ \\
\hline Prior pulmonary embolism & $12 \%$ & $2 \%$ & 0.016 \\
\hline Pulmonary embolism post procedure & $4 \%$ & $1 \%$ & 0.25 \\
\hline Charlson comorbidity index & $3.3 \pm 2.6$ & $2.2 \pm 1.8$ & 0.006 \\
\hline Infection upon generator replacement & $17 \%$ & $29 \%$ & 0.24 \\
\hline Lead types for defibrillator explants: & & & 0.90 \\
\hline Right atrial & $38 \%$ & $34 \%$ & \\
\hline Right ventricular & $46 \%$ & $50 \%$ & \\
\hline Left ventricular & $16 \%$ & $16 \%$ & \\
\hline \multicolumn{4}{|l|}{ Lead types for pacemaker explants: } \\
\hline Right atrial & $48 \%$ & $47 \%$ & 0.83 \\
\hline Right ventricular & $52 \%$ & $53 \%$ & \\
\hline Left ventricular & $0 \%$ & $0 \%$ & \\
\hline Use of powered laser system & - & $60 \%$ & - \\
\hline Success of all lead removal & $96 \%$ & $94 \%$ & 1.00 \\
\hline Other infections: & $12 \%$ & $4 \%$ & 0.08 \\
\hline Urinary infection & 1 & 4 & \\
\hline Pneumonia & 0 & 3 & \\
\hline Septic emboli & 2 & 1 & \\
\hline Epidural abscess & 0 & 2 & \\
\hline Arthritic infection & 0 & 2 & \\
\hline Osteomyelitis & 0 & 1 & \\
\hline Post-surgical antibiotic duration [days] & $5.2 \pm 1.0$ & $3.2 \pm 1.9$ & $<0.01$ \\
\hline Complications of antibiotic therapy: & $12 \%$ & $4 \%$ & 0.08 \\
\hline Acute interstitial nephritis & 2 & 4 & \\
\hline Altered mental status & 1 & 0 & \\
\hline Clostridium difficile colitis & 0 & 5 & \\
\hline Drug fever & 0 & 1 & \\
\hline Rash & 0 & 1 & \\
\hline Elevated liver enzymes & 0 & 1 & \\
\hline Renal failure & 0 & 1 & \\
\hline
\end{tabular}




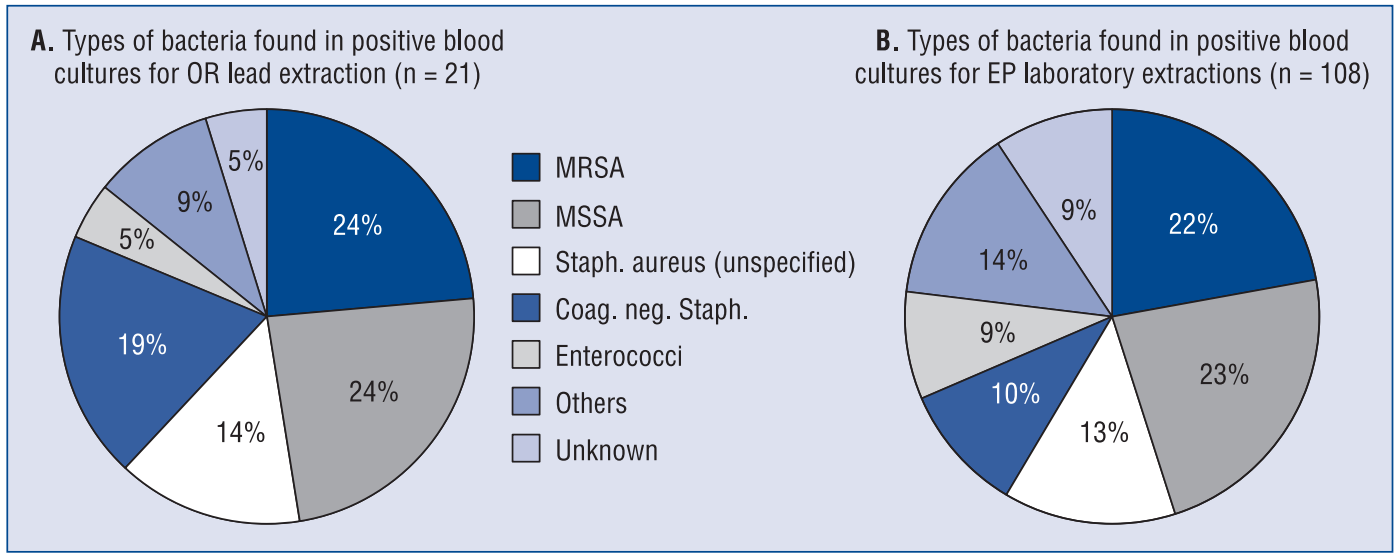

Figure 1. Bacteria recovered from blood cultures of patients undergoing lead extraction in the operating room via open thoracotomy (OR) (A) versus percutaneously in the electrophysiology (EP) laboratory (B). Staphylococcal infection where sensitivity data was not available is in white.

Table 2. Mortality and non-lethal complication rates for the two study groups.

\begin{tabular}{lccc}
\hline Adverse events & $\begin{array}{c}\text { Open } \\
\text { thoracotomy }\end{array}$ & $\begin{array}{c}\text { Percutaneous } \\
\text { techniques }\end{array}$ & P \\
\hline Deaths after 30 days & $4 / 24(17 \%)$ & $15 / 329(5 \%)$ & 0.036 \\
Deaths after 6 months & $8 / 24(33 \%)$ & $45 / 329(14 \%)$ & 0.020 \\
Deaths after 1 year & $10 / 24(42 \%)$ & $61 / 329(19 \%)$ & 0.012 \\
Non-lethal complications during procedure & $0 / 24(0.0 \%)$ & $6 / 329(2.0 \%)$ & 0.99 \\
Non-lethal complications 30 days post procedure & $2 / 24(8 \%)$ & $22 / 329(7 \%)$ & 0.31 \\
Hospital length of stay [days] & $23 \pm 15$ & $12 \pm 9$ & $<0.001$ \\
\hline
\end{tabular}

Table 3. Univariate predictors of death 1 year after the index procedure.

\begin{tabular}{lccc}
\hline Variable & Died (n = 71) & Alive (n = 281) & P \\
\hline Age [years] & $75 \pm 12$ & $68 \pm 13$ & $<0.001$ \\
Gender (men) & $66 \%$ & $77 \%$ & 0.06 \\
Ejection fraction [\%] & $34 \pm 14$ & $40 \pm 16$ & 0.023 \\
Creatinine [mg/dL] & $2.0 \pm 1.4$ & $1.4 \pm 1.0$ & $<0.001$ \\
Coronary artery disease & $82 \%$ & $61 \%$ & 0.001 \\
Diabetes mellitus & $51 \%$ & $34 \%$ & 0.012 \\
ORS width [ms] & $137 \pm 35$ & $135 \pm 41$ & 0.74 \\
Positive blood cultures or vegetations & $61 \%$ & $37 \%$ & $<0.001$ \\
Open-thoracotomy extraction & $14 \%$ & $5 \%$ & 0.012 \\
Implantable cardioverter-defibrillator explanted & $59 \%$ & $50 \%$ & 0.21 \\
Charlson comorbidity index & $3.1 \pm 2.2$ & $2.0 \pm 1.7$ & $<0.001$
\end{tabular}

ted CIED system and found that the mortality rate is about $17 \%$ at 30 days after the index procedure. This is in keeping with the results of a smaller study by Wilhelm et al. [21] examining the outcome of 8 patients with infected pacemaker systems undergoing OR in which a $12.5 \% 30$-day mortality rate was reported. Camboni et al. [22] reported a lower rate of death 30 days after surgery (9\%) in 21 patients receiving OR. It is worth mentioning, however, that this latter study included a significant number of non-infected patients who were therefore less sick compared to our patient population. 


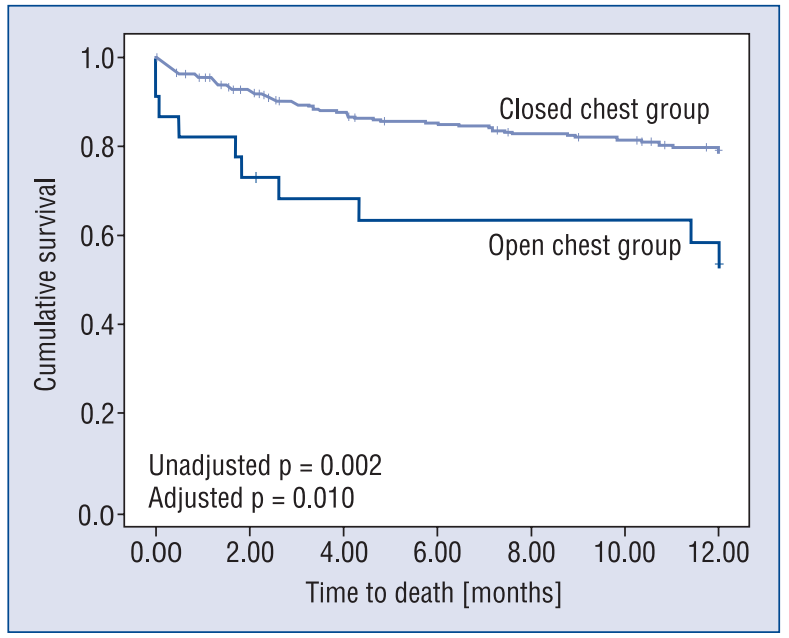

Figure 2. Kaplan-Meier curves showing the survival of patients with cardiac implantable electronic devices infection after lead extraction in both the open-thoracotomy (OR) (open chest) and percutaneously (EP) (closed chest) study groups.

The mortality and morbidity associated with CIED system extraction for infectious indications using closed-chest techniques has been previously examined. Grammes et al. [17] reported on the feasibility of explanting such infected CIED systems in 100 patients with documented large vegetations by transesophageal echocardiography. The 30-day mortality in that cohort of patients was $10 \%$, which is higher than the $5 \%$ rate we report in our present population. The difference in mortality is most likely attributable to the fact that our patients were less sick than the ones included in the study by Grammes et al. [17] as only $19 \%$ of our patients had documented vegetations.

The mode of death in patients who expired within 30 days of the index procedure was different between our two study groups. Whereas half of the OR patients died of cardiogenic shock presumably induced by the invasive surgery performed with general anesthesia and cardiopulmonary bypass, most of the EP-treated patients died of sepsis and multi-organ failure. These data add some insight into the shortcomings of each of these two treatment strategies.

\section{Limitations of the study}

Our study has some limitations. First, it is a single-center study that may not represent the experience at other institutions. The presence of local expertise in both surgical and percutaneous lead extractions is a prerequisite for making clinical choices at any institution and for providing surgical support in the event of complications or failure of the percutaneous approach. Second, our study is retrospective and may therefore include biases, such as performance of concomitant surgeries in the open-chest group that could not be eliminated despite the statistical adjustment of multivariate analyses. In addition, the size of the two study groups was very different, which may introduce further bias. We have, however, included all patients undergoing lead extraction for infectious indications to minimize potential sources of bias. Therefore, the numbers included in each of the study group represent the overall clinical practice at our institution. Multivariate models were also used to adjust for unbalanced variables between the study groups. Lastly, because of incomplete documentation in the operative and original implantation notes, the fixation type of extracted leads and the number of coils on extracted defibrillator leads could not be ascertained in this study.

\section{Conclusions}

In conclusion, infected CIED patients undergoing open-chest lead extraction are sicker and have higher mortality rates compared to those undergoing percutaneous extraction. Randomized, prospective data are needed to determine whether the procedural strategy for lead extraction can impact the outcome of CIED infected patients.

Table 4. Cox proportional hazard predictor of death 1 year after index procedure.

\begin{tabular}{lcccc}
\hline & $\mathbf{P}$ & Odds ratio & \multicolumn{2}{c}{ 95\% confidence interval } \\
\cline { 3 - 5 } & & & Lower & Upper \\
\hline Open chest (vs. closed chest) & 0.010 & 2.60 & 1.26 & 5.37 \\
Gender (women vs. men) & 0.08 & 0.64 & 0.39 & 1.05 \\
Age (1 year increment) & $<0.001$ & 1.04 & 1.02 & 1.06 \\
Charlson comorbidity index & 0.003 & 1.17 & 1.05 & 1.29 \\
\hline
\end{tabular}




\section{Conflict of interest: Evan Adelstein - Research grants from SJM, MDT; Samir Saba - Research grants from SJM, MDT, BSC.}

\section{References}

1. Moss AJ, Zareba W, Hall WJ et al.; Multicenter Automatic Defibrillator Implantation Trial II Investigators. Prophylactic implantation of a defibrillator in patients with myocardial infarction and reduced ejection fraction. N Engl J Med, 2002; 346: 877-883.

2. Buxton A, Lee K, DiCarlo L, Gold M et al. Electrophysiologic testing to identify patients with coronary artery disease who are at risk for sudden death. N Engl J Med, 2000; 342: 1937-1945.

3. The Antiarrhythmics versus Implantable Defibrillators (AVID) Investigators. A comparison of antiarrhythmic-drug therapy with implantable defibrillators in patients resuscitated from near-fatal ventricular arrhythmias. N Engl J Med, 1997; 337: 1576-1583.

4. Epstein AE, DiMarco JP, Ellenbogen KA et al. ACC/AHA/HRS 2008 Guidelines for Device-Based Therapy of Cardiac Rhythm Abnormalities: a report of the American College of Cardiology/ /American Heart Association Task Force on Practice Guidelines (Writing Committee to Revise the ACC/AHA/NASPE $2002 \mathrm{Gu}$ ideline Update for Implantation of Cardiac Pacemakers and Antiarrhythmia Devices) developed in collaboration with the American Association for Thoracic Surgery and Society of Thoracic Surgeons. J Am Coll Cardiol, 2008; 51: e1-e62.

5. Zhan C, Baine W, Sedrakyan A, Steiner C. Cardiac device implantation in the United States from 1997 through 2004: A population-based analysis. J Gen Intern Med, 2008; 23: 13-19.

6. Peterson PN, Daugherty SL, Wang Y et al.; the National Cardiovascular Data Registry. Gender differences in procedure-related adverse events in patients receiving implantable cardioverter-defibrillator therapy. Circulation, 2009; 119: 1078-1084.

7. Cabell $\mathrm{CH}$, Heidenreich $\mathrm{PA}, \mathrm{Chu} \mathrm{VH}$ et al. Increasing rates of cardiac device infections among Medicare beneficiaries: 1990-1999. Am Heart J, 2004; 147: 582-586.

8. Voigt A, Shalaby A, Saba S. Rising rates of cardiac rhythm management device infections in the United States: 1996 through 2003. J Am Coll Cardiol, 2006; 48: 590-591.

9. Voigt A, Shalaby A, Saba S. Continued rise in rates of cardiovascular implantable electronic device infections in the United States: Temporal trends and causative insights. Pacing Clin Electrophysiol, 2010; 33: 414-419.

10. Chua JD, Wilkoff BL, Lee I, Juratli N, Longworth DL, Gordon SM. Diagnosis and management of infections involving implantable electrophysiologic cardiac devices. Ann Intern Med, 2000; 133: 604-608.

11. Love CJ, Wilkoff BL, Byrd CL et al. Recommendations for extraction of chronically implanted transvenous pacing and defibrillator leads: Indications, facilities, training. Pacing Clin Electrophysiol, 2000; 23: 544-551.

12. Sohail MR, Uslan DZ, Khan AH et al. Management and outcome of permanent and implantable cardioverter-defibrillator infections. J Am Coll Cardiol, 2007; 49: 1851-1859.

13. Deharo JC, Quatre A, Mancini J et al. Long-term outcomes following infection of cardiac implantable electronic devices: A prospective matched cohort study. Heart, 2012; 98: 9724-9731.

14. Chamis AL, Peterson GE, Cabell CH et al. Staphylococcus aureus bacteremia in patients with permanent pacemakers or implantable cardioverter defibrillators. Circulation, 2001; 104: 1029-1033.

15. Chiu WS, Nguyen D. Pacemaker lead extraction in pacemaker endocarditis with lead vegetation: usefulness of transesophageal echocardiography. Can J Cardiol, 1998; 14: 87-89.

16. Meier-Ewert H, Gray ME, John R. Endocardial pacemaker or defibrillator leads with infected vegetations: A single-center experience and consequences of transvenous extraction. Am Heart J, 2003; 146: 339-344.

17. Grammes J, Schulze C, Al-Bataineh M et al. Percutaneous pacemaker and implantable cardioverter-defibrillator lead extraction in 100 patients with intracardiac vegetations defined by transesophageal echocardiogram. J Am Coll Cardiol, 2010; 55: 886-894.

18. Nguyen KT, Neese P, Kessler DJ. Successful laser-assisted percutaneous extraction of four pacemaker leads associated with large vegetations. Pacing Clin Electrophysiol, 2000; 23: 1260-1262.

19. Wilkoff BL, Love CJ, Byrd CL et al. Heart Rhythm Society; American Heart Association. Transvenous lead extraction: Heart Rhythm Society expert consensus on facilities, training, indications, and patient management: This document was endorsed by the American Heart Association (AHA). Heart Rhythm, 2009; 6: 1085-1104.

20. Charlson ME, Pompei P, Ales KL, MacKenzie CR. A new method of classifying prognostic comorbidity in longitudinal studies: development and validation. J Chronic Dis, 1987; 40: 373-383.

21. Wilhelm MJ, Schmid C, Hammel D et al. Cardiac pacemaker infection: surgical management with and without extracorporal circulation. Ann Thorac Surgery, 1997; 64: 1707-1712.

22. Camboni D, Wollmann CG, Loher A, Gradaus R, Scheld HH, Schmid C. Explantation of implantable defibrillator leads using open heart surgery or percutaneous techniques. Ann Thorac Surg, 2008; 85: 50-55. 